\title{
SECOND GENERATION HIGH GRADIENT QUADRUPOLES FOR THE LHC INTERACTION REGIONS ${ }^{1}$
}

\begin{abstract}
T. Sen, J. Strait and A.V. Zlobi
Abstract

Conceptual designs of large-aperture high-gradient $\mathrm{Nb}_{3} \mathrm{Sn}$ quadrupoles, suitable for use in a second generation LHC interaction region, are presented. A quadrupole with a $90 \mathrm{~mm}$ coil aperture and the same 200 $\mathrm{T} / \mathrm{m}$ gradient as in the current LHC IR is technically feasible and would allow $\beta^{*}=0.25 \mathrm{~m}$ to be achieved, doubling the LHC luminosity.
\end{abstract}

\section{INTRODUCTION}

The first generation of low-beta quadrupoles for the LHC IR inner triplets based on NbTi superconductor is being developed by KEK (MQXA) and Fermilab (MQXB) in collaboration with CERN [1,2]. These magnets provide a nominal field gradient of $200 \mathrm{~T} / \mathrm{m}$ at the high luminosity insertions with $70-\mathrm{mm}$ coils, and operate at $1.9 \mathrm{~K}$ under high radiation-induced heat load. The magnets will allow achieving the nominal luminosity of $10^{34} \mathrm{~cm}^{-2} \mathrm{~s}^{-1}$. To reach the ultimate luminosity of $2.5 \times 10^{34} \mathrm{~cm}^{-2} \mathrm{~s}^{-1}$ optics changes are required. The IR quadrupole parameters such as nominal gradient, field quality (dynamic aperture) and available physical aperture are the main factors limiting the machine performance. Given that the lifetime of the first generation IR quadrupoles is limited to 5-7 years, the second generation of IR quadrupoles have to be developed with the goal to achieve the ultimate luminosity.

The gradient and aperture of the $1^{\text {st }}$ generation IR quads are set by the use of NbTi superconductor. Further increase of the field gradient in the large bore quadrupoles requires new superconductors. Recent progress in the development of $\mathrm{Nb}_{3} \mathrm{Sn}$ strand with higher critical current density, critical magnetic field and critical temperature as well as advances of the $\mathrm{Nb}_{3} \mathrm{Sn}$ magnet technology allow considering it for $2^{\text {nd }}$ generation IR quadrupoles.

\section{BEAM OPTICS}

The luminosity can be doubled by lowering $\beta^{*}$ by a factor of two. This change has a significant influence on the beam optics, including the beam size in the IRs, linear and nonlinear chromaticity generated by the IRs, dynamic aperture and the long-range beam-beam interactions. We examine some of these issues assuming two different models of the second generation quadrupoles.

The first model has a $70 \mathrm{~mm}$ aperture and a $275 \mathrm{~T} / \mathrm{m}$ gradient. The larger gradient allows the quadrupole length to be shortened while keeping the same integrated strength. The quadrupoles in the triplet except for Q1 may

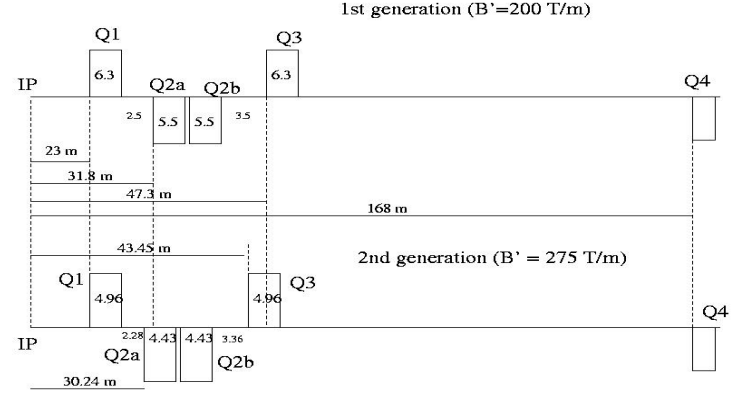

Figure 1: The layout with the present quadrupoles and a possible layout with these higher gradient quadrupoles.

be moved closer to the IP which reduces the peak $\beta$ values. Figure 1 shows the layout with the present quadrupoles and a possible layout with the higher gradient quadrupoles. All quadrupoles starting from Q4 are unchanged. The optics is matched to the entrance of Q7. Figure 2 shows the $\beta$-functions from the IP $\beta^{*}=0.25$ $\mathrm{m}$ to Q7. Table 1 shows the quadrupole lengths and locations and the peak $\beta$-functions within them corresponding to the layout shown in Figure 1. The peak $\beta$-functions are reduced e.g. in Q3, from $\beta \approx 9400 \mathrm{~m}$ to $\beta \approx 7900 \mathrm{~m}$ in the $2^{\text {nd }}$ relative to the $1^{\text {st }}$ generation layout. Besides increasing the physical aperture, the dynamic aperture with stronger quadrupoles would improve provided the field quality is the same.

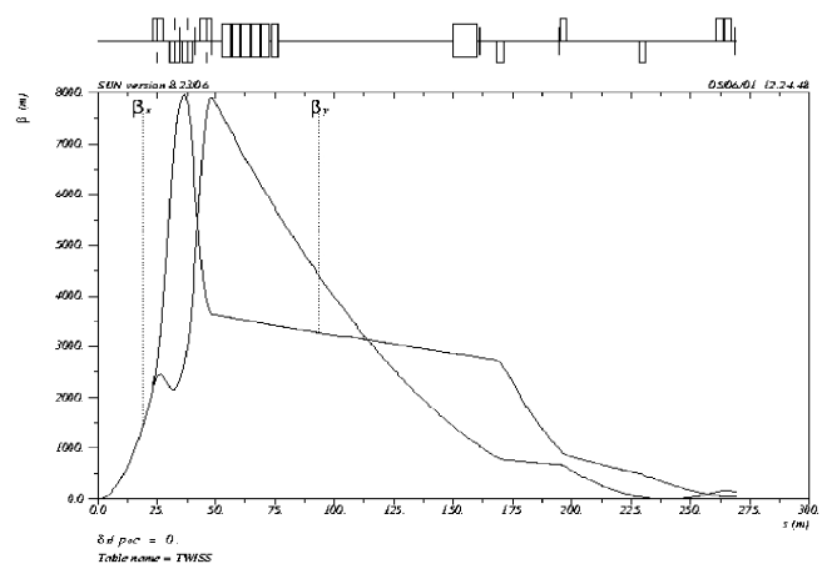

Figure 2: The $\beta$-functions with the $275 \mathrm{~T} / \mathrm{m}$ quadrupoles in the inner triplet and $\beta^{*}=0.25 \mathrm{~m}$.

The long range interactions have a strong influence on the dynamic aperture. Therefore when $\beta^{*}$ is decreased by a factor of two, the crossing angle should be increased by

\footnotetext{
${ }^{1}$ Work supported by the U.S. Department of Energy

E-mail: tsen@fnal.gov, strait@fnal.gov, zlobin@fnal.gov
} 
Table 1: Parameters of the $1^{\text {st }}$ and $2^{\text {nd }}$ generation $275 \mathrm{~T} / \mathrm{m}$ quadrupoles.

\begin{tabular}{|l|l|l|l|l|l|l|}
\hline Quad & \multicolumn{2}{|c|}{ Length [m] } & \multicolumn{2}{|c|}{$\mathrm{L}(\mathrm{IP} \rightarrow \mathrm{Q})[\mathrm{m}]$} & \multicolumn{2}{c|}{ Peak $\beta[\mathrm{m}]$} \\
& \multicolumn{1}{|c|}{$1^{\text {st }}$} & $2^{\text {nd }}$ & $1^{\text {st }}$ & $2^{\text {nd }}$ & $1^{\text {st }}$ & $2^{\text {nd }}$ \\
\hline Q1 & 6.3 & 4.96 & 23.0 & 23.0 & 4541 & 4000 \\
Q2a & 5.5 & 4.43 & 31.8 & 30.24 & 9202 & 7779 \\
Q2b & 5.5 & 4.43 & 38.3 & 35.67 & 9344 & 7918 \\
Q3 & 6.3 & 4.96 & 47.3 & 43.45 & 9411 & 7914 \\
Q4 & 3.0 & 3.0 & 168.0 & 168.0 & 2967 & 2596 \\
\hline
\end{tabular}

$\sqrt{2}$ to keep the same distance between the beams (in units of the rms beam size) at parasitic collision points. Thus if the full crossing angle at $\beta^{*}=0.50 \mathrm{~m}$ is $300 \mu \mathrm{rad}$, the crossing angle at $\beta^{*}=0.25 \mathrm{~m}$ needs to be $424 \mu \mathrm{rad}$. Higher luminosity will most likely require thicker absorbers in the $2^{\text {nd }}$ generation triplet quadrupoles. If we increase the thickness from $3 \mathrm{~mm}$ to $5 \mathrm{~mm}$ in Q2a, Q2b and Q3, the minimum geometrical acceptance with $\beta^{*}=0.25 \mathrm{~m}$, crossing angle $=424 \mu \mathrm{rad}$, is $4.3 \sigma$ using the procedure in [3]. This value is unacceptably small. With the absorber thickness kept at $3 \mathrm{~mm}$ and the geometrical acceptance and beam separations kept at their present values of $7 \sigma$ and $9.4 \sigma$ respectively, the smallest allowed $\beta^{*}$ is $0.41 \mathrm{~m}$. This only increases the luminosity by $22 \%$. To be more aggressive we could choose the smallest allowable geometric acceptance, $5.5 \sigma$, and the thinnest beam tube feasible, $1.5 \mathrm{~mm}$. Then a $9 \sigma$ beam separation with $\beta^{*}=0.25 \mathrm{~m}$ is possible. However this leaves no margin for error in machine operation and may lead to unacceptably large energy deposition in the quadrupoles.

The other option is to use $90 \mathrm{~mm}$ aperture magnets with the same gradient as in the $1^{\text {st }}$ generation. In this case no optics redesign is necessary. The aperture is large enough that we can increase the absorber thickness to $5 \mathrm{~mm}$ and still have geometrical acceptance of $7 \sigma$ with $\beta^{*}=0.25 \mathrm{~m}$ and a beam separation of $9.4 \sigma$. Figure 3 shows the physical aperture and the $7 \sigma$ beam envelope in the triplets with this configuration. In calculations of the geometric aperture, the assumed closed orbit errors are $4 \mathrm{~mm}$, tolerance on the physical aperture of the absorbers is 0.7 $\mathrm{mm}$ and of the quadrupoles is $1.6 \mathrm{~mm}$. The second option of the $90 \mathrm{~mm}$ aperture quadrupoles with gradients of 200 $\mathrm{T} / \mathrm{m}$ is clearly the superior option on the path to higher luminosity.

\section{QUADRUPOLE DESIGN}

Conceptual designs of $2^{\text {nd }}$ generation IR quadrupoles were developed for the two above cases. The crosssections were optimized using ROXIE [4]. To reduce cost and time in replacing the IR quadrupoles their design should be compatible with the available cryogenics and electrical hardware. In particular, the magnet operation current should not exceed $15 \mathrm{kA}$, which is determined by the capacity of current leads. The quadrupoles will also be

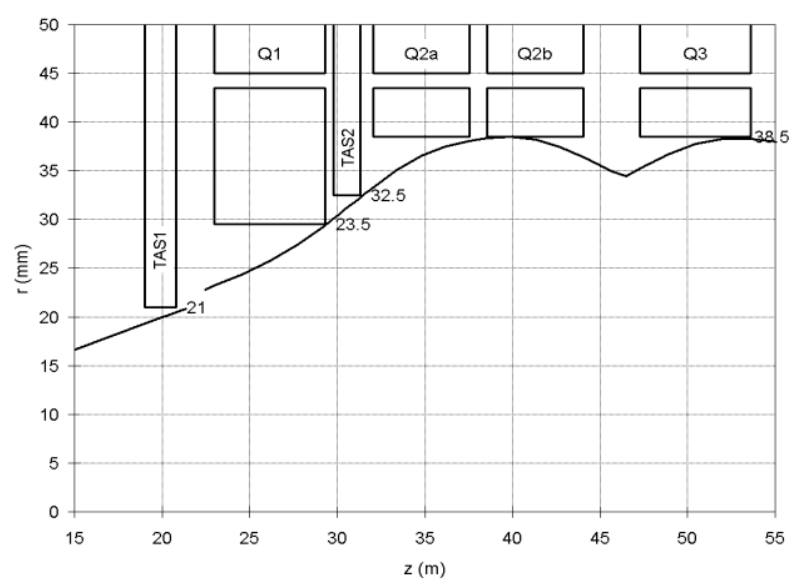

Figure 3: The aperture in the inner triplet with $90 \mathrm{~mm}$ aperture quadrupoles, $\beta^{*}=0.25 \mathrm{~m}$, beam separation $=9.4 \sigma$.

operating in superfluid helium with the nominal temperature of $1.95 \mathrm{~K}$.

Figure 4 shows cross-sections of the quadrupole coils with 70-mm (IRQ70) and 90-mm (IRQ90) bore diameter. Both designs are based on two-layer shell-type coils and cold iron yoke and use the same Rutherford-type cable made of $42 \mathrm{Nb}_{3} \mathrm{Sn}$ strands, $0.7 \mathrm{~mm}$ in diameter. The cable width is $15.50 \mathrm{~mm}$, the small edge is $1.118 \mathrm{~mm}$ and the large edge is $1.350 \mathrm{~mm}$. It is insulated with a $200-\mu \mathrm{m}$ thick high-temperature insulation. Coil fabrication procedure uses the wind and react technique being developed at Fermilab for the $\mathrm{Nb}_{3} \mathrm{Sn}$ dipoles [5].

The coils are supported by the iron yoke and the thick stainless steel skin. The yoke inner diameter is $160(180)$ $\mathrm{mm}$ for the IRQ70 (IRQ90). The $12 \mathrm{~mm}$ thick metal spacers fill the space between the coil and yoke. The iron outer diameter and final cross-section will be determined later based on iron saturation effects, fringe fields, and the cold mass interface with the existing MQX cryostat. The parameters of the IRQ70 and IRQ90 are summarized in Table 2 and compared with those of the MQXB (Fermilab's design of MQX).

The maximum field gradient at $1.9 \mathrm{~K}$ vs. the critical current density $\mathrm{J}_{\mathrm{C}}$ of the $\mathrm{Nb}_{3} \mathrm{Sn}$ strand at $12 \mathrm{~T}$ and $4.2 \mathrm{~K}$ is shown in Figure 5. To provide a $275 \mathrm{~T} / \mathrm{m}$ gradient in the

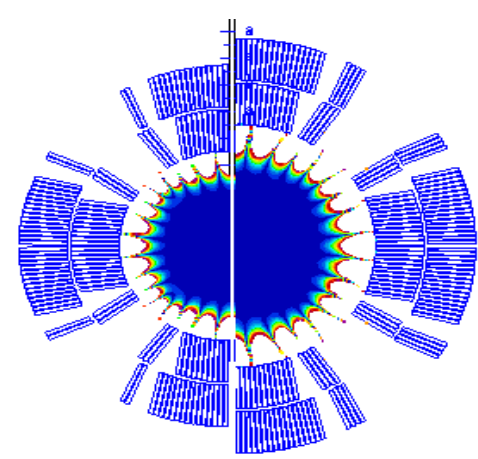

Figure 4: IRQ coil cross-sections:

left - 70-mm quadrupole, right - 90-mm quadrupole. 
Table 2: Quadrupole design parameters.

\begin{tabular}{|l|c|c|c|}
\hline & MQXB & IRQ70 & IRQ90 \\
\hline Superconductor & $\mathrm{NbTi}$ & $\mathrm{Nb}_{3} \mathrm{Sn}$ & $\mathrm{Nb}_{3} \mathrm{Sn}$ \\
\hline Bore diameter, mm & 70 & 70 & 90 \\
\hline Yoke ID, mm & 185.12 & 160.00 & 180.00 \\
\hline Number of turns & 120 & 120 & 148 \\
\hline $\mathrm{L}, \mathrm{mH} / \mathrm{m}$ & 3.5 & 3.17 & 5.07 \\
\hline $\mathrm{G} / \mathrm{I} @ \mathrm{I}_{\text {nom }}, \mathrm{T} / \mathrm{m} / \mathrm{kA}$ & 18.09 & 17.43 & 15.02 \\
\hline $\mathrm{G}_{\text {nom }}, \mathrm{T} / \mathrm{m}$ & 205 & 275 & 205 \\
\hline $\mathrm{I}_{\text {nom }}, \mathrm{kA}$ & 11.33 & 15.78 & 13.65 \\
\hline
\end{tabular}

70-mm bore quadrupole with the critical current margin of $20 \%$ (similar to MQX) $\mathrm{J}_{\mathrm{C}}$ of the superconductor in the coil must be at least $2.6 \mathrm{kA} / \mathrm{mm}^{2}$. Assuming a $10 \%$ strand critical current degradation the required nominal $\mathrm{J}_{\mathrm{C}}$ of the virgin strands is $2.9 \mathrm{kA} / \mathrm{mm}^{2}$, which is not available now. In contrary, the nominal gradient of $200 \mathrm{~T} / \mathrm{m}$ with $20 \%$ critical current margin in the $90-\mathrm{mm}$ bore quadrupole requires the $\mathrm{Nb}_{3} \mathrm{Sn}$ strands having the critical current density only $2.2 \mathrm{kA} / \mathrm{mm}^{2}$. Such strands are commercially produced at the present time [6].

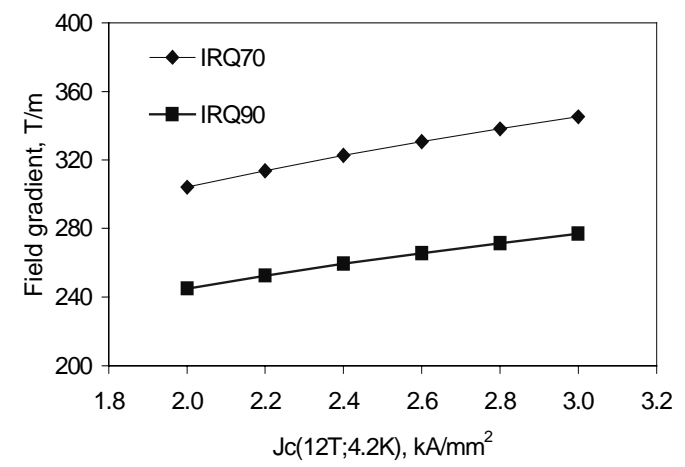

Figure 5: Short sample limit for the IRQ70 and IRQ90 at the operation temperature of $1.9 \mathrm{~K}(\mathrm{Cu}: n o n C u=1.2: 1)$.

The design low-order harmonics for the quadrupole central field are reported in Table 3 at $17 \mathrm{~mm}$ bore radius and are compared with those for the MQXB. The systematic component of $b_{6}$ at injection related to the persistent current effect is large due to the large magnetization of available $\mathrm{Nb}_{3} \mathrm{Sn}$ strands [6]. However, it will be significantly reduced by reducing the effective filament diameter or using a simple passive correction based on thin iron strips [7]. Eddy current effects will be minimized using a small filament twist pitch and optimizing the crossover resistance in the cable. The iron saturation effect on $b_{6}$ at collision will be corrected by optimizing the size and position of holes in the iron yoke.

Table 3: Geometrical harmonics

\begin{tabular}{|c|c|c|c|}
\hline & MQXB & IRQ70 & IRQ90 \\
\hline $\mathrm{b}_{6}$ & -0.013 & 0.00016 & 0.00029 \\
\hline $\mathrm{b}_{10}$ & -0.001 & -0.00007 & 0.00002 \\
\hline $\mathrm{b}_{14}$ & -0.0011 & -0.0065 & -0.00019 \\
\hline
\end{tabular}

Table 4: Harmonic RMS spread, sigma

\begin{tabular}{|c|c|c|c|c|c|c|}
\hline & \multicolumn{2}{|c|}{ MQXB } & \multicolumn{2}{c|}{ IRQ70 } & \multicolumn{2}{c|}{ IRQ90 } \\
\hline $\mathrm{n}$ & $\mathrm{a}_{\mathrm{n}}$ & $\mathrm{b}_{\mathrm{n}}$ & $\mathrm{a}_{\mathrm{n}}$ & $\mathrm{b}_{\mathrm{n}}$ & $\mathrm{a}_{\mathrm{n}}$ & $\mathrm{b}_{\mathrm{n}}$ \\
\hline 3 & 0.28 & 0.26 & 1.56 & 1.46 & 0.947 & 0.890 \\
\hline 4 & 0.37 & 0.08 & 0.735 & 0.771 & 0.356 & 0.375 \\
\hline 5 & 0.15 & 0.07 & 0.386 & 0.380 & 0.151 & 0.148 \\
\hline 6 & 0.05 & 0.17 & 0.169 & 0.173 & 0.052 & 0.054 \\
\hline 7 & 0.03 & 0.03 & 0.078 & 0.076 & 0.019 & 0.019 \\
\hline 8 & 0.01 & 0.00 & 0.035 & 0.032 & 0.007 & 0.006 \\
\hline 9 & 0.01 & 0.00 & 0.014 & 0.014 & 0.002 & 0.002 \\
\hline 10 & 0.00 & 0.01 & 0.006 & 0.006 & 0.0008 & 0.0007 \\
\hline
\end{tabular}

The calculated harmonics RMS spread for block radial and azimuthal random displacements within $\pm 50 \mu \mathrm{m}$, is reported in Table 4 and compared with the RMS spread measured for the last five MQXB short models [8]. Analysis shows that the systematic low order harmonics could be provided on the same level as in the MQXB in both IRQ70 and IRQ90 but only IRQ90 provides the random harmonics variation comparable with the MQXB.

\section{CONCLUSIONS}

The conceptual designs of the second generation IR quadrupoles with $70-\mathrm{mm}$ and $90-\mathrm{mm}$ bore diameter and $\mathrm{Nb}_{3} \mathrm{Sn}$ coils have been developed and analysed. The 90 $\mathrm{mm}$ bore quadrupole provides the nominal field gradient of $200 \mathrm{~T} / \mathrm{m}$ with sufficient critical current margin using $\mathrm{Nb}_{3} \mathrm{Sn}$ strand commercially produced at the presant time and field quality comparable with the field quality in MQXB. Magnet physical and dynamic aperture allows reducing the $\beta^{*}$ and increasing the beam crossing angle required for achieving the ultimate luminosity in LHC.

\section{REFERENCES}

[1]. N. Andreev et al., IEEE Trans. Appl. Supercon., v.11, No. 1, March 2001, p. 1558.

[2]. T. Shintomi et al., IEEE Trans. Appl. Supercon., v.11, No. 1, March 2001, p. 1562.

[3] J.B. Jeanneret, T. Risselada, "Geometrical aperture in LHC at injection," LHC Project Note 66, September 1996.

[4]. S. Russenschuck, "A Computer Program for the Design of Super-conducting Accelerator Magnets", CERN AC/95-05 (MA), September 1995.

[5]. D.R. Chichili et al., IEEE Trans. Appl. Supercon., v. 11, No. 1, March 2001, p. 2160.

[6]. E. Barzi et al., IEEE Trans. Appl. Supercon., v. 11, No. 1, March 2001, p. 3595.

[7]. V.V. Kashikhin, A.V. Zlobin, IEEE Trans. Appl. Supercon., v.11, No. 1, March 2001, p. 2058.

[8]. N. Andreev et al., Trans. Appl. Supercon., v.11, No. 1, March 2001, p. 1566. 